\title{
Measurement of U1tra-Heavy Cosmic Rays at a Lunar Base
}

M.H. Salamon [1], P.B. Price [2], and G. Tarle [3]

[1] Physics Department, University of Utah, Salt Lake City, UT 84112

[2] Physics Department, University of California at Berkeley, Berkeley, CA 94720

[3] Physics Department, University of Michigan, Ann Arbor, MI 48109

A wealth of information regarding cosmic ray synthesis and propagation is contained in the ultra-heavy $(Z>60)$ cosmic ray abundances; to extract this information, however, requires a detector capable of acquiring large statistics for these rare particles, as well as a charge resolution adequate to separate neighboring charge peaks at very large $Z$. A large, passive surface array of nuclear-track-detecting glass plates would meet these requirements. These glass plates could be periodically processed and analyzed for tracks at a lunar base, then melted/annealed for reuse in a continuously recycled detector array.

\section{U1tra-Heavy Cosmic Rays:}

An accurate measurement of the elemental abundances of the ultra-heavy $(Z>60)$ cosmic rays would contribute substantially to our understanding of the origin, acceleration, and propagation of cosmic rays within our Galaxy. In particular, a high statistics measurement of the abundances of the actinide elements in the cosmic rays, along with the possible detection of transuranics, would provide definitive answers to questions that have remained outstanding since the discovery of the cosmic ray heavy-nucleus component in 1948 [1].

One of the foremost questions regards the source of the nuclear cosmic rays. Data from the HEAO-3 [2], HEAO-C [3], and Ariel 6 [4] satellites show that for $\mathrm{Z}<60$ the galactic cosmic ray (GCR) elemental distribution is consistent with the assumption of a source whose elemental abundances are the same as that of our solar system (SS). Observed deviations from unity of these (normalized) GCR/SS abundance ratios can be attributed to ionization selection effects, viz., the probability of initial acceleration (hence selection) of an ion in a source is dependent upon its first ionization potential (FIP). The striking similarity of the SEP(solar energetic particle)/SS elemental abundance ratios to the GCR/SS ratios suggests [5] that nascent GCR's originate in stellar chromospheres, where temperatures are such that the element's FIP determines ionization fraction and ejection to the stellar corona. 
These ions then reach GCR energies via first-order Fermi acceration within shock waves that are produced by neighboring supernovae or stellar winds. A collorary of this picture is that ss abundances (as determined by meteorites and solar photospheric measurements) are essentially the same as those of the present local interstellar medium (ISM).

The above picture, however, does not disallow variations in isotopic and elemental composition in various source regions due to ongoing chemical evolution within the ISM, and these are in fact seen. Isotopic GCR data from IMP [6] and ISEE [7] satellites show significant deviations in certain isotopic ratios (such as ${ }^{22} \mathrm{Ne} /{ }^{20} \mathrm{Ne}$ ), indicating that additional nuclear processing has occurred for some fraction of the GCR. The data for GCR's of $z>$ 60, from HEAO-C and Ariel 6, suggest that there may be a significant enhancement of $r$-process [8] elements in this component [3], so that we may be directly observing the products of (possibly recent) explosive nucleosynthesis.

This raises the possibility that a fraction of the ultraheavy GCR's may be freshly synthesized nuclei from recent supernovae, accelerated to cosmic ray energies by the supernova shocks. Confirmation of this would give us strong evidence for the role of shocks in nuclear GCR acceleration, and would provide an observational window to nucleosynthetic environments perhaps different from our own. A high-statistics measurement of the abundances of the GCR actinides would provide a definitive answer to this question. Figure 1 (taken from Ref. 9) shows the abundances of the actinide elements from $\mathrm{Z}=90$ to 96 as a function of time after explosive nucleosynthesis. Since the propagation lifetime of GCR actinides should be less than $10^{7}$ yr $[10]$, it is clear that the GCR actinide composition from freshly synthesized material would be quite distinct from that of old $\left(>10^{9} \mathrm{yr}\right)$ ISM material. Due to their differing lifetimes, the $U / T h$ ratio, equal to 0.60 in $S S$ material [11], would be greater than unity in fresh $r$-process material. In addition, the observation of comparable fluxes of transuranic elements, such as $\mathrm{Cm}$ whose halflife is $1.6 \times 10^{7} \mathrm{yr}$, would be an unambiguous signature of fresh $r$-process enhancement of the GCR's.

To make these observations, however, requires very large detector area and collection time, as well as unprecedented charge resolution to obtain clean charge separation in the actinide region. To put this in perspective, we note that a grand total of 4 actinides were detected by the HEAO-C and Ariel 6 instruments; the (superior) charge resolution of the HEAO-C instrument was considerably worse than 1 charge unit in the actinide region, making impossible the identification of individual actinides. To achieve an accuracy of 108 in the U/Th ratio, over $400 \mathrm{U}$-Th ions must be detected (with a charge resolution of 0.5 charge units or better); 
it is evident that satellites with electronic payloads cannot easily be scaled up in size to meet these collection requirements.

A detector that is capable of cleanly separating even- and odd- $\mathrm{Z}$ elements in the $\mathrm{Pt}-\mathrm{Pb} / \mathrm{sub}-\mathrm{Pt}-\mathrm{Pb}$ region would also do much to improve our understanding of the $C R$ propagation pathlength distribution (PLD) for pathlengths on the order of $1 \mathrm{~g} / \mathrm{cm}^{2}$ and $1 \mathrm{ess}$, since the nuclear interaction length of these nuclei in the interstellar medium is on the order of $1 \mathrm{~g} / \mathrm{cm}^{2}$. The simplest model of CR propagation, the leaky box with a negative exponential PLD, fails to account for the observed abundances of $\mathrm{CR}$ secondaries (spallation products of heavier cosmic-ray parents) in the $\mathrm{Li}-\mathrm{B}$ and sub-Fe regions. To obtain agreement with these data, an energydependent truncation of the smallest pathlengths $\left(<1 \mathrm{~g} / \mathrm{cm}^{2}\right)$ in the PLD must be made [12]. This supports the hypothesis of a nested leaky box model [13], in which newly accelerated $\mathrm{CR}^{\prime} s$ must travel through significant grammage before leaving their site of acceleration. Thus the $0-1 \mathrm{~g} / \mathrm{cm}^{2}$ region of the PLD can very likely yield clues to the structure of the astrophysical sites within which CR's are born. Element-resolved abundance measurements of the Pt$\mathrm{Pb} / \mathrm{sub}-\mathrm{Pt}-\mathrm{Pb}$ region are the best way to constrain this important region of the PLD; again, high statistics are required to obtain the necessary accuracy in the secondary/primary ratios, which argues for detector sizes far larger than those previously flown. We note that for these measurements it is imperative that there be as little upstream mass as possible; even a fraction of a gram per squarecentimeter will cause significant fragmentation of the ultra-heavy CR flux, contaminating the astrogenic secondary CR flux. Thus balloons, whose payloads typically have float altitudes of several $\mathrm{g} / \mathrm{cm}^{2}$, are unsultable for this type of measurement, leaving orbiting facilities, and now a lunar base, as ideal deployment sites.

Finally, we note that a high-statistics measurement of the $z$ $>60 \mathrm{CR}$ elemental abundances may yield unexpected surprises. If superheavy $(Z>100)$ elements are in fact produced in supernovae and have halflives in excess of $10^{7}$ years, then in the event that $a$ fraction of the nuclear CR's are comprised of fresh $r$-process material, a detector such as that described below might provide the first evidence for the existence of an island of nuclear stability beyond the transuranics. Speculative particles such as quark nuggets [14] may be searched for as well.

\section{A Lunar-Based Heavy Nucleus Collector (LBHNC):}

The recent discovery [15] of the extraordinary properties of certain phosphate glasses as nuclear track detectors [16] has made possible the construction of an extremely simple and powerful detector of ultra-heavy cosmic rays of $\mathrm{Z}>60$. When an ionizing projectile passes through a generic nuclear-track-detecting medium, it creates a latent track, which is a region of damage (typically Angstroms in radius) about the projectile's trajectory. Subsequent 
to its exposure, the material is chemically etched in a corrosive liquid, which preferentially removes material from the damaged region, creating a visible track or cone whose geometric parameters, determined by microscopy, determine the ratio of projectile charge to velocity [16]. A detector composed of several thin layers of this material measures this ratio in successive layers as the ionizing particle is slowing; the variation of track etch rate with depth in the material uniquely identifies the charge of the particle and its incident energy.

Conceptually, an ultra-heavy CR detector comprised of nuclear-track-detecting material, such as sheets of certain types of glass or plastic, is very simple. A given detector module would consist of a sufficient number of thin sheets of (e.g.) glass stacked together to ensure that a particle's charge be unambiguously identified by the method described above; nuclear interactions cause a loss of signal, so that it would be important to keep the glass sheets as thin as possible. To minimize temporal variations in temperature the modules would be thermally isolated with thermal standoffs and multilayer insulation. Total collection area could be increased as desired by the addition of modules to the experiment site. No power would be required for such a detector, as it would be completely passive during its acquisition of latent tracks. Thus the achievement of a total detector area on the order of $10^{2} \mathrm{~m}^{2}$ would be quite straightforward; with a collection time of a few years, given the absence of a significant magnetic field on the Moon, the number of observed $C R$ actinides would be increased by over 2 orders of magnitude above the present world's total (assuming solar system abundances for the CR actinides).

One nuclear-track-detecting glass in particular, BP-1 [17], has remarkable immunity to the problems that must be faced by any long-duration experiment composed of nuclear track detectors that is deployed in space. Those problems include the fading of latent tracks over exposure periods of months or years at even fairly low temperatures [18], the variation of the sensitivity of the detecting medium with temperature [19] and oxygen pressure [20], and the growth of latent track reactivity with time [21]. BP-1 glass, produced by Schott, suffers no fading of latent tracks up to the test limit of 5 months at $50{ }^{\circ} \mathrm{C}[22]$, ensuring that no detectable fading would occur at a lunar base site even over a several year period. The temperature dependence of the glass sensitivity is also remarkably small at $-20^{\circ} \mathrm{C}$ (the designed operating temperature of the passive detector) with a fractional shift in the subsequent track etch rate of $-1 \times 10^{-3}$ per degree Centigrade of temperature at the time of track creation. In addition, unlike many other trackdetecting media, the response of $\mathrm{BP}-1$ in vacuum is the same as in air, so that no pressure vessels (with their unavoidable mass overburden) need be employed. There also is no measurable variation of latent track reactivity with time in BP-1 glass. 
The charge resolution of BP-1 glass is unequalled in the high-Z regime. Figure 2 shows data on the fragmentation of $1 \mathrm{GeV} / \mathrm{u}$ $\mathrm{Au}(\mathrm{Z}-79)$ lons produced by Lawrence Berkeley Laboratory's Bevalac. A total of 5 sheets (10 surfaces) of glass sufficed to yield a charge resolution of 0.06 charge units (note that the ordinate is logarithmic)! The underlying physical reason [23] for such extraordinary charge resolution is that a latent track in glass is created only by electrons that receive low momentum transfers from the ionizing projectile (and thus deposit their energy close to the particle's trajectory); their number is very large, so that the statistical variation of damage within the track is negligible. This is in constrast to the energy deposited far away from the track by high-energy electrons (delta-rays); the number of high-energy electrons is far smaller, and thus statistical fluctuations are of importance; detectors which are sensitive to the energy deposited by these electrons (glass is not) w1ll therefore suffer larger fluctuation in response.

The actual charge resolution of the LBHNC detector is a complicated function of the intrinsic resolution given above and the ability to uniquely fit response curves of individual elements to the measured track etch rate vs. stack depth of any given event. Figure 3 shows the expected charge resolution versus CR energy as determined by a Monte Carlo calculation for a module composed of 14 sheets of $0.2 \mathrm{~cm}$ thick BP-1 glass. It is seen that a majority of the ultra-heavy $C R$ events will have their charge determined to an accuracy of $<0.3$ charge units.

A lunar site is arguably the ideal location for an HNC detector. The absence of a magnetic field means that lower energy ultra-heavy CR's are not screened from the detector, as they would be for a detector in a low-inclination orbit about the Earth. Assuming solar system abundances, the integral flux of CR actinides of energy $>0.85 \mathrm{GeV} / \mathrm{u}$ (ensuring adequate range within the glass) would be $-1 \mathrm{U} / \mathrm{m}^{2}-\mathrm{yr}$ and $2 \mathrm{Th} / \mathrm{m}^{2}-\mathrm{yr}$; a $100 \mathrm{~m}^{2}$ detector of mass -8000 $\mathrm{kg}$ would therefore collect close to 1000 actinides in a $3 \mathrm{yr}$ period. This would be more than adequate to determine the U/Th ratio to < 108; such a detector would also be sensitive to transuranics even if the fresh $r$-process component of the GCR's comprised only a few percent of total CR flux.

After its 3-year exposure, the glass could be returned to Earth for chemical processing and automated track microscopy and analysis. Alternatively, if a chemical etching facility and an automated track imaging system were to be established at the lunar base, the glass culd be harvested, analyzed, then either annealed or remelted at the lunar base, then placed back onto the lunar surface for additional exposure; in effect, the LBHNC detector could be indefinitely recycled at no additional payload cost. 


\section{References:}

[1] P.S. Freier et al., Phys. Rev. 74, 213 (1948).

[2] P. Goret et al., Proc. 18th ICRC (Bangalore) 9, 139 (1983), and references therein.

[3] W.R. Binns et al., to appear in Ap. J., Nov.15,1989.

[4] P.H. Fowler et a1., Ap. J. 314, 739 (1987).

[5] J.P. Meyer, Ap. J. Supp1. 57, 173 (1985).

[6] M. Garcia-Munoz, J. Simpson, and J.P. Wefel, Ap. J. Lett. 232, L95 (1979).

[7] M.E. Wiedenbeck and D.E. Greiner, Phys. Rev. Lett. 46, 682 (1981).

[8] E.M. Burbidge, G.R. Burbidge, W.A. Fowler, and F. Hoyle, Rev. Mod. Phys . 29, 547 (1957).

[9] J.B. Blake and D.N. Schramm, Astrophys. and Sp. Sci. 30, 275 (1974).

[10] T.G. Guzik et al., Proc. 19th ICRC (La Jolla) 2, 76 (1985).

[11] A.G.W. Cameron, in Essays in Nuclear Astrophysics, ed. C.A. Barnes et al., Cambridge University Press (1982).

[12] J.P. Wefel, in Genesis and Propagation of Cosmic Rays, p.1, eds. M.M. Shapiro and J.P. Wefel (Reidel, Dordrecht, 1988).

[13] R. Cowsik and L.W. Wilson, Proc. 14th Inter. Cosmic Ray Conf. 2. 659 (Munich, 1975).

[14] E. Witten, Phys. Rev. D30, 272 (1984),

[15] P.B. Price et al., Nucl. Instr. Meth. B21, 60 (1987).

[16] R.L. Fleischer, P.B. Price, and R.M. Walker, Nuclear Tracks in Solids, University of California Press (1975).

[17] Shicheng Wang et al., Nucl. Instr. Meth. (in press, 1989).

[18] M.H. Salamon, P.B. Price, and J. Drach, Nucl. Instr. Meth. B17, 173 (1986).

[19] A. Thompson et al., Proc. 11th Inter. Conf. on Solid State Nuclear Track Detectors (Bristol, England, 1981).

[20] J. Drach et al., Nucl. Instr. Meth. B23, 367 (1987).

[21] J. Drach and P.B. Price, Nuc1. Instr. Meth. B28, 275 (1987).

[22] A.J. Westfall et al., to be published.

[23] G. Tarle, S.P. Ahlen, and P.B. Price, Nature 293, 556 (1981). 


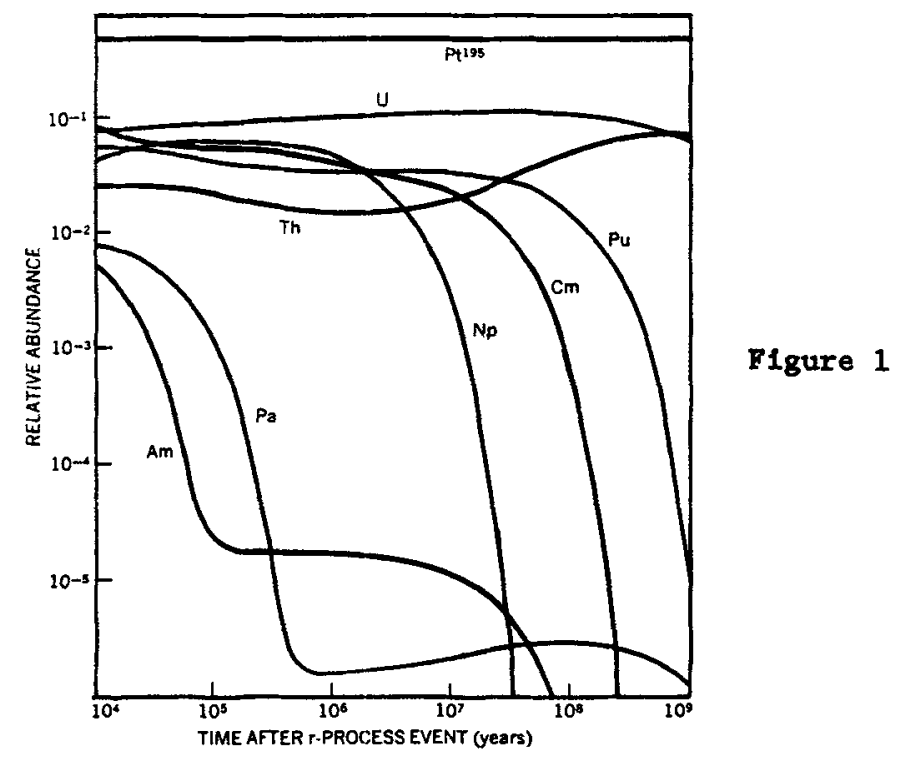

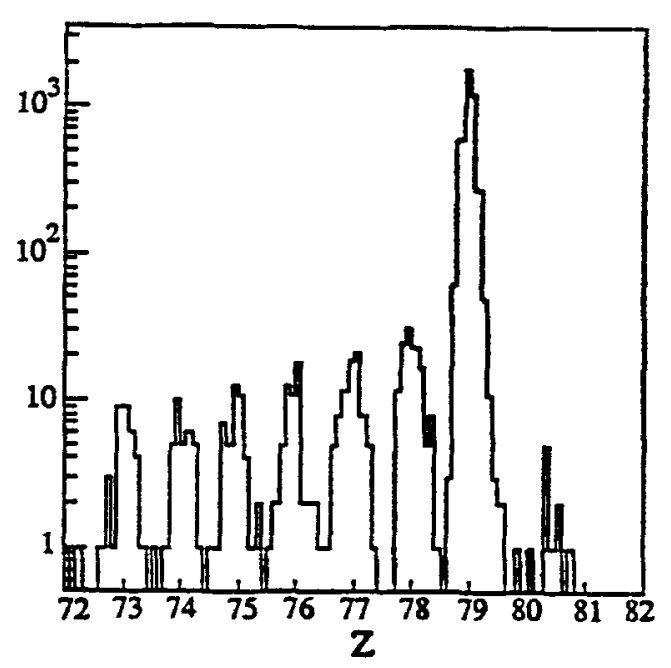

Figure 2

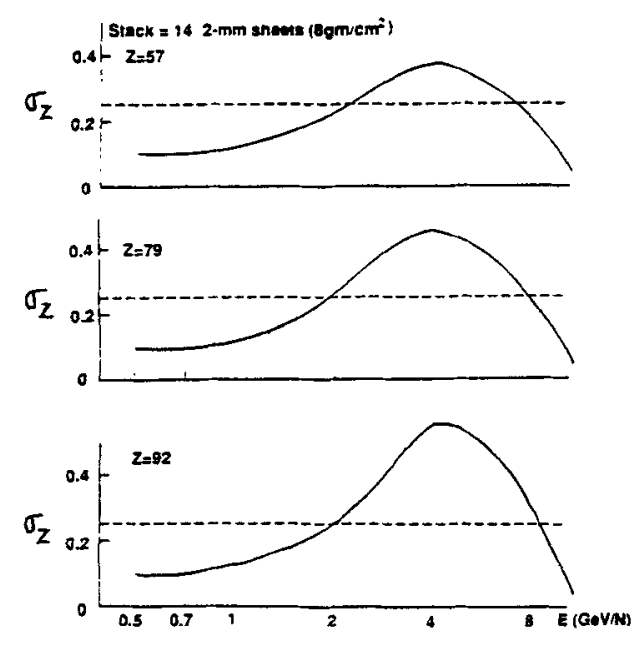

Figure 3 